\title{
Phylogenetic Typing of Bacillus anthracis Isolated in Japan by Multiple Locus Variable-Number Tandem Repeats and the Comprehensive Single Nucleotide Polymorphism
}

\author{
Akiko OKUTANI ${ }^{1) * *}$, Tsuyoshi SEKIZUKA ${ }^{2) \#}$, Bazartseren BOLDBAATAR ${ }^{1,3)}$, Akio YAMADA ${ }^{1,3)}$, Makoto KURODA ${ }^{2)}$ \\ and Satoshi INOUE ${ }^{1,3)}$ \\ ${ }^{1)}$ Department of Veterinary Science and ${ }^{2)}$ Pathogen Genomics Center, National Institute of Infectious Diseases, 1-23-1 Toyama,
Shinjuku-ku, Tokyo 162-8640 and ${ }^{3)}$ The United Graduate School of Veterinary Science, Gifu University, 1-1 Yanagido, Gifu 501-1193,
Japan
}

(Received 12 May 2009/Accepted 9 September 2009)/Published online in J-STAGE 13 November 2009)

ABSTRACT. Twelve strains of Bacillus anthracis isolated in Japan were subjected to multiple locus variable-number tandem repeats analysis using 25 marker loci (MLVA25). The results showed that Japanese strains could be divided into two distinct genetic clusters, A3a and A3b. By using newly devised comprehensive single nucleotide polymorphisms (SNPs) analysis, Japanese strains were also divided into two groups. The results obtained by the MLVA25 and plasmids SNP analysis well coincided, indicating that both methods were highly sensitive to discriminate $B$. anthracis strains. These results suggested that MLVA25 had sufficient discrimination power to identify $B$. anthracis at the strain level, and that MLVA25 as well as comprehensive SNPs analysis could facilitate further studies of $B$. anthracis strains including Japanese and other Asian strains.

KEY WORDS: Bacillus anthracis, MLVA, phylogeny, SNP.

J. Vet. Med. Sci. 72(1): 93-97, 2010

Anthrax caused by Bacillus anthracis, spore forming agent, is one of the most severe zoonoses, which poses serious threats to both public and animal health. Although anthrax has been almost eradicated from Japan except some sporadic human [10], or animal cases [8,9], there are growing concern over potential use of $B$. anthracis as a bioterrorism agent. In addition, various animal products imported from all over the world including those countries where anthrax is enzootic may become vehicles of anthrax from abroad as exemplified by an event recently reported in the UK [2]. It appears, therefore, important to have a sensitive and specific method for identifying $B$. anthracis isolated from humans and animals as well as from the environments at the strain level.

Since $B$. anthracis shows very limited genetic diversity [4], identification of $B$. anthracis strains requires methods which have high discrimination power. Multiple locus variable-number tandem repeats analysis (MLVA) has been used for identification of $B$. anthracis at the strain level. The number of target loci increased from eight (MLVA8) in the initial report [5] to twenty five (MLVA25) in the most recently published work [6]. Single nucleotide polymorphisms (SNPs) analysis also became a powerful genetic tool to differentiate $B$. anthracis strains [12]. A large numbers of canonical SNPs scattered throughout the whole genome were proved to be regarded as strain-specific markers that enables us to distinguish geographically and genetically

\footnotetext{
* Correspondence to: Okutani, A., Department of Veterinary Science, National Institute of Infectious Diseases, 1-23-1 Toyama, Shinjuku-ku, Tokyo 162-8640 Japan. e-mail:okutani@nih.go.jp

\# These authors contributed equally to this work.
}

diverse isolates [14].

In this study, we have attempted to clarify the phylogenetic relationship of $B$. anthracis strains isolated in Japan with those from other parts of the world by MLVA25 as well as comprehensive SNP analysis. B. anthracis strains used in this study are listed in Table 1 . These strains were kindly provided by several prefectural livestock hygiene service centers. BA102, 103, 108, 109 and 111 strains were isolated from dairy or beef cattle while BA104, 105 [1] and 107 [8] were isolated in the sporadic incidents of pigs which occurred in 1982. Neither the infectious routes nor sources have been identified. All the isolates were positive for 2 pathogenic plasmids: $181.7 \mathrm{~kb}$ pXO1 encoding the protective antigen, lethal factor and edema factor [11] and $96.2 \mathrm{~kb}$ pXO2 encoding the capsule operon [7]. They were also gamma phage sensitive, penicillin and ciprofloxacin sensitive, non-hemolytic on sheep blood agar (SBA) and nonmotile.

Table 1. Bacillus anthracis strains isolated in Japan

\begin{tabular}{cccc}
\hline Strain number & Isolated place & Isolated animal & Isolated year \\
\hline BA102 & Miyagi & dairy cattle & 1983 \\
BA103 & Miyagi & beef cattle & 1991 \\
BA104 & Shizuoka & pig & 1982 \\
BA105 & Shizuoka & pig & 1982 \\
BA106 & Okinawa & unknown & 1956 \\
BA107 & Okinawa & pig & 1982 \\
BA108 & Shiga & dairy cattle & 1987 \\
BA109 & Mie & dairy cattle & 1970 \\
BA110 & Mie & unknown & 1967 \\
BA111 & Okayama & dairy cattle & 1985 \\
BA113 & Okayama & human & unknown \\
BA115 & Shizuoka & unknown & unknown \\
\hline
\end{tabular}


Table 2. Genetical information of strains used for SNP analysis

\begin{tabular}{|c|c|c|c|c|c|c|c|}
\hline Strain name & pXO1 & $\mathrm{pXO} 2$ & chromosome & SNP analysis ${ }^{\text {a) }}$ & MLVA $25^{\mathrm{b})}$ & canSNP typing ${ }^{\mathrm{c}}$ & Accession Number \\
\hline A0193 & + & + & + & A1a & A1a & A.Br.WNA & NZ_ABKF00000000 \\
\hline A0174 & + & $\left.-{ }^{d}\right)$ & + & A1a & & & NZ_ABLT00000000 \\
\hline USA6153 & + & + & + & A1a & & & NZ_AAER00000000 \\
\hline Australia 94 & + & + & + & A3a & A3a & A.Br.Aust94 & NZ_AAES00000000 \\
\hline BA104 & This study & This study & N.A.e) & A3a & A3a & & \\
\hline A2012 & + & + & + & $\mathrm{A} 3 \mathrm{~b}$ & & & NZ_AAAC00000000 \\
\hline Ames & - & - & + & $\mathrm{A} 3 \mathrm{~b}$ & $\mathrm{~A} 3 \mathrm{~b}$ & & NC_003997 \\
\hline Ames Ancestor & + & + & + & $\mathrm{A} 3 \mathrm{~b}$ & & & NC_007530 \\
\hline BA103 & This study & This study & N.A. & $\mathrm{A} 3 \mathrm{~b}$ & $\mathrm{~A} 3 \mathrm{~b}$ & & \\
\hline Sterne & + & - & + & $\mathrm{A} 3 \mathrm{~b}$ & $\mathrm{~A} 3 \mathrm{~b}$ & & NC_005945 \\
\hline A0389 & + & + & + & $\mathrm{A} 3 \mathrm{~b}$ & & A.Br.001/002 & NZ_ABLB00000000 \\
\hline A0488 & + & + & + & A4 & A4 & & NZ_ABJC00000000 \\
\hline Vollum & + & + & + & A4 & A4 & & NZ_AAEP00000000 \\
\hline A0442 & + & + & + & B1 & & B.Br.Kruger & $\mathrm{ABK} G 01000000$ \\
\hline Kruger B & + & + & + & B1 & B1 & B.Br.Kruger & NZ_AAEQ00000000 \\
\hline A 0465 & + & + & + & B2 & B2 & B.Br.CNEVA & NZ_ABLH00000000 \\
\hline CNEVA-9066 & + & + & + & B2 & B2 & B.Br.CNEVA & NZ_AAEN00000000 \\
\hline A1055 & - & + & + & $\mathrm{C}$ & $\mathrm{C}$ & & NZ_AAEO00000000 \\
\hline Tsiankovskii-I & + & + & + & new & & & NZ_ABDN00000000 \\
\hline
\end{tabular}

a) This study.

b) Refered by Lista et al. [6] and Fig. 1 .

c) Refered by Van Ert et al. [14].

d) Plasmid genome sequence is not available.

e) Not available.

Table 3. The observed and expected fragment sizes by fragment analysis and the number of repeats on 25 loci of MLVA in 12 B. anthracis strains isolated in Japan

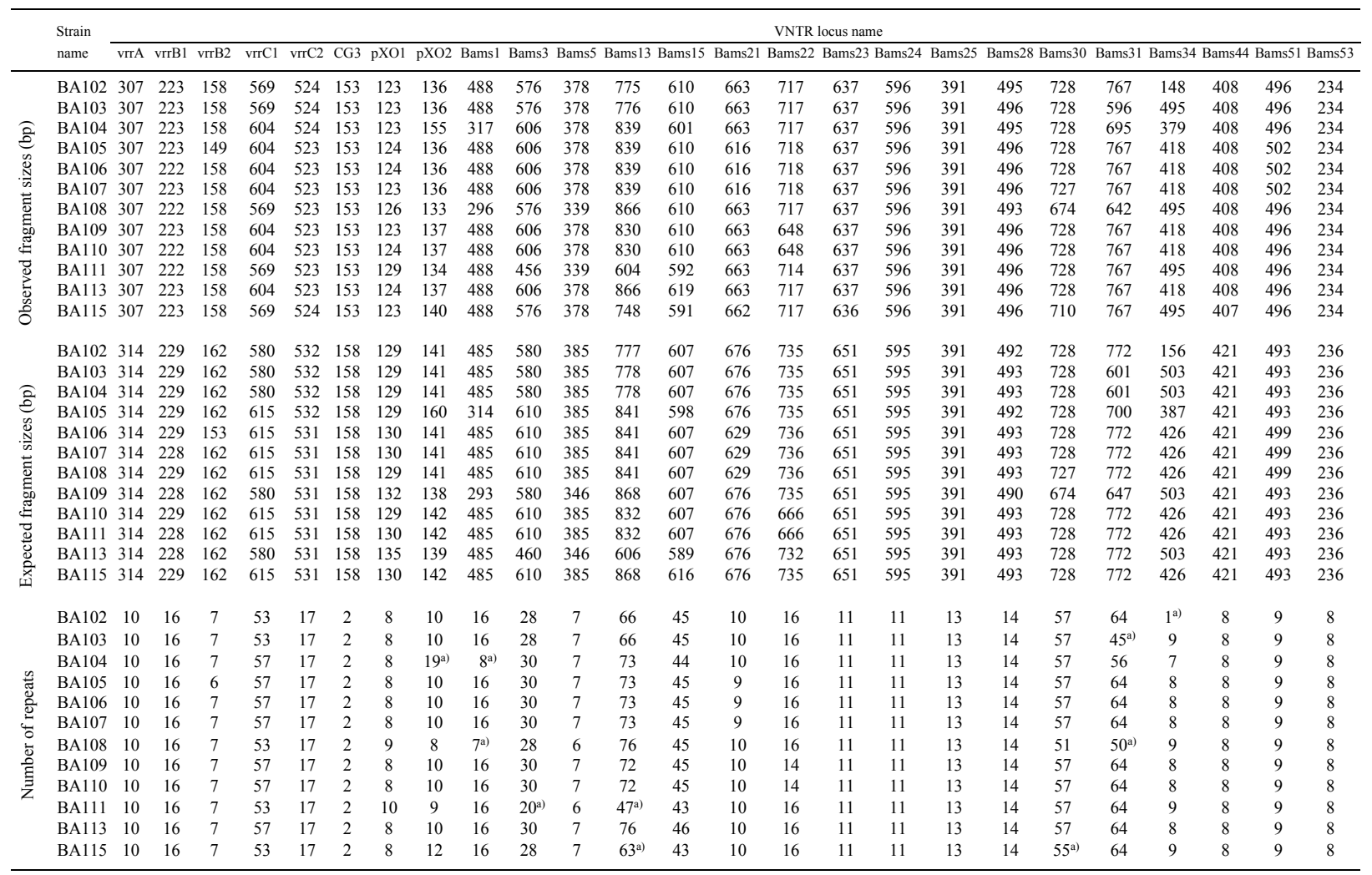

a) new allele. 


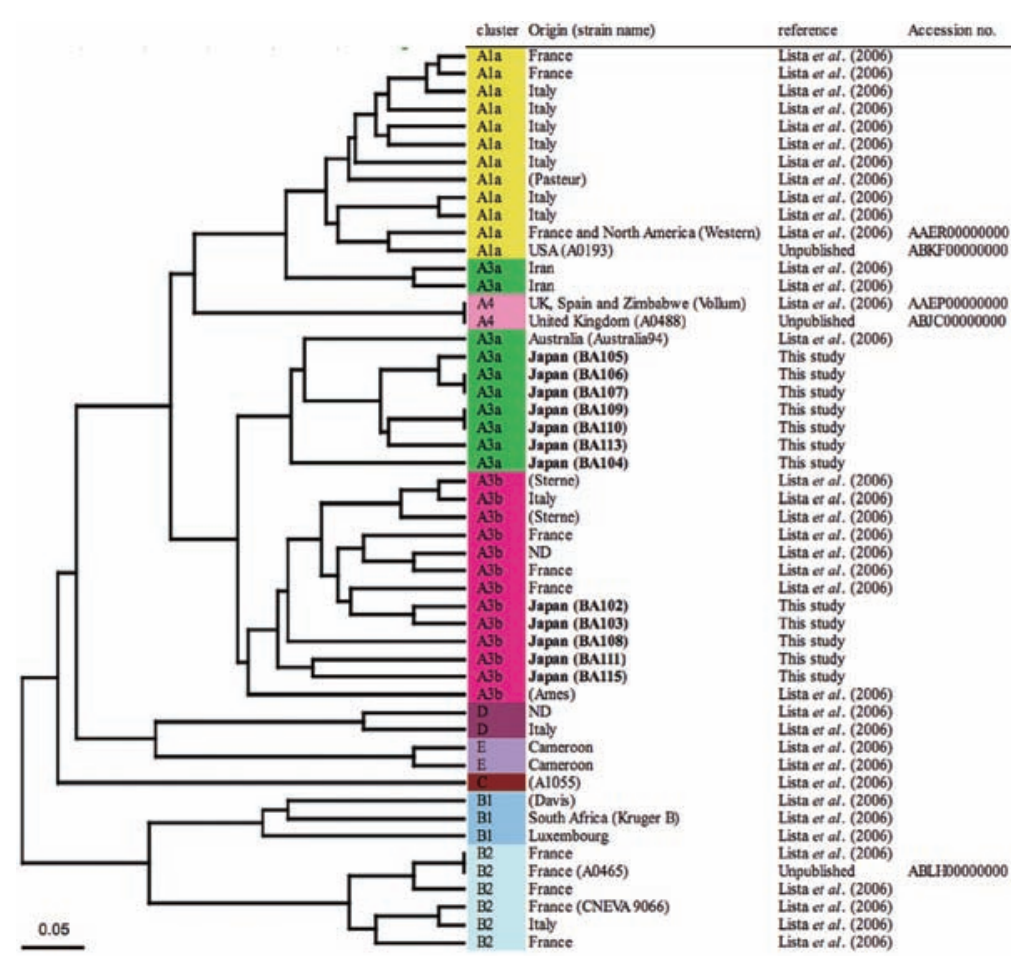

Fig. 1. Dendrogram constructed according to the MLVA25 of 51 B. anthracis strains including 12 Japanese isolates. The tree was drawn by UPGMA method using PHYLIP version 3.6 and Tree View versom1.6.6. "Cluster" refers to the clustering as proposed by Lista et al. [6]. The origin and strain (when known) names are indicated; ND, not defined.

(A)

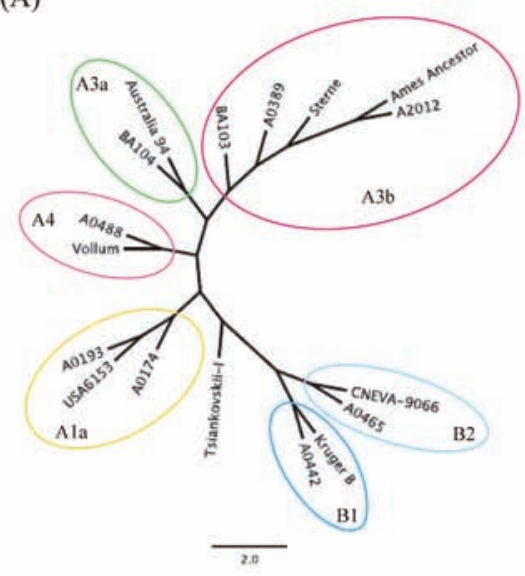

(B)

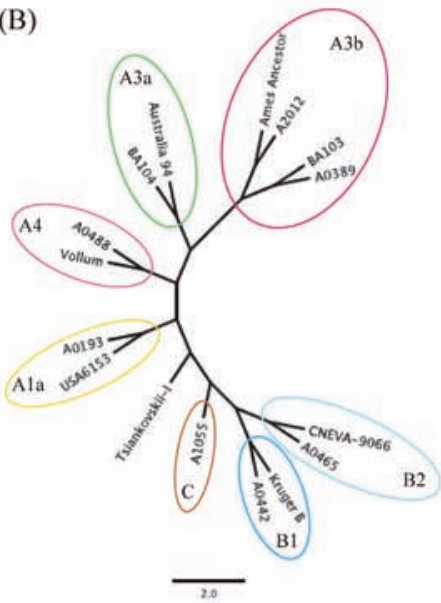

(C)

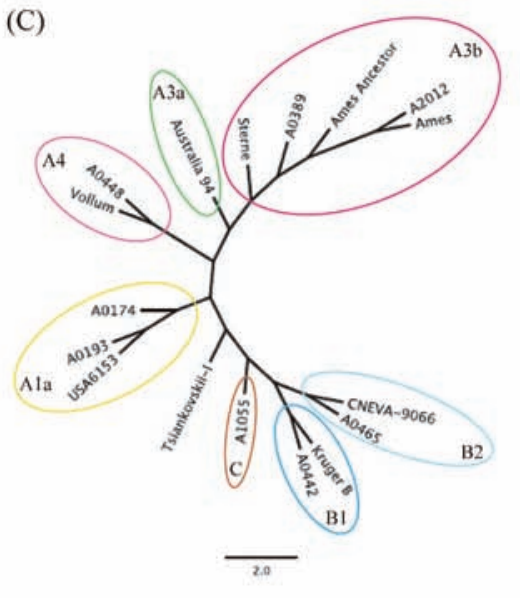

Fig. 2. Unrooted phylogenetic tree of B. anthracis strains constructed based on SNPs found in the nucleotide sequences of $\mathrm{pXO1}$ (A), pXO2 (B) and chromosomal DNA (C). The trees were drawn using two computer programs, Clustal X and FigTree. The BA103 and BA104 were included in the SNP analysis for the plasmid DNAs but not for the chromosomal DNAs. The clusters by MLVA25 are also shown.

MLVA25 was performed according to the method reported by Lista et al. with some modifications as mentioned below. Fluorescent dyes, 6-FAM, VIC and PET were used for primer labeling (Applied Biosystems Inc.). The bacterial cells cultured overnight at $37^{\circ} \mathrm{C}$ on SBA plates
(Eiken chemical, Tokyo, Japan) were lysed with $1 \mathrm{mg} / \mathrm{ml}$ each of lysozyme and achromopeptidase (WAKO chemical, Tokyo, Japan) at $37^{\circ} \mathrm{C}$ for $30 \mathrm{~min}$ followed by further digestion with $1 \mathrm{mg} / \mathrm{m} l$ proteinase $\mathrm{K}$ at $56^{\circ} \mathrm{C}$ overnight. Genomic DNA was extracted with phenol-chloroform and recovered 
by ethanol precipitation. PCR amplification was performed with Phusion High-Fidelity DNA polymerase (Finnzymes, Espoo, Finland) using following condition; initial denaturation at $98^{\circ} \mathrm{C}$ for $2 \mathrm{~min}$, followed by 30 cycles of denaturation at $98^{\circ} \mathrm{C}$ for $10 \mathrm{sec}$, annealing at $55^{\circ} \mathrm{C}$ for $15 \mathrm{sec}$, extension at $72^{\circ} \mathrm{C}$ for $30 \mathrm{sec}$, and further extension at $72^{\circ} \mathrm{C}$ for $5 \mathrm{~min}$. Annealing temperature was reduced to $50^{\circ} \mathrm{C}$ for Bams28, 30, 3144 and 53 amplification. After capillary electrophoresis of the PCR products using the Applied Biosystems 3730 xl DNA Analyzer (Applied Biosystems Inc.), the fragment sizes were calculated by Peak Scanner software version 1.0 (Applied Biosystems Inc.) using GENESCAN 1200 LIZ Size standard (Applied Biosystems Inc.) as internal size standard. Since we do not have any $B$. anthracis strains used by Lista et al. in their MLVA25 analysis, the exact numbers of the tandem repeats of 25 loci of the BA103 strain were determined by the nucleotide sequencing. The number of repeats of the respective loci of other Japanese strains was deduced by the comparing the capillary electrophoregram with that of BA103.

By performing MLVA25, ten new alleles on 7 loci were identified (Table 3). The phylogenetic tree constructed with 25 VNTR loci by combining our data together with those reported by Lista et al. as well as those found in NCBI database (A0193, A0488 and A0465) revealed that Japanese isolates were divided into two distinct clusters. BA104, 105, $106,107,109,110$ and 113 were clustered in the A3a, together with the Australia94 strain, while 5 other strains were included in the A3b cluster with the Ames strain (Fig. 1).

The entire nucleotide sequences of $\mathrm{pXO} 1$ and $\mathrm{pXO} 2$ plasmids of BA103 and BA104 strains were determined by primer waking methods by $3730 \mathrm{xl}$ DNA Analyzer with Big Dye Terminator v 3.1 Cycle sequencing kit (Applied Biosystems Inc.) with the PCR products, up to $\sim 8 \mathrm{~kb}$, amplified with Platinum Taq DNA polymerase High Fidelity (Invitrogen Japan, Tokyo, Japan). Obtained sequences were assembled and edited with Phred/Phrap/Consed [3]. SNPs on $\mathrm{pXO} 1$ and $\mathrm{pXO} 2$ plasmid DNA among $B$. anthracis strains shown in Table 2 were extracted by the SNPs Finder program [13]. All SNPs were concatenated to hypothetical nucleotide sequences for each strain, and the sequences were aligned using Clustal X version 1.83. An unrooted phylogenetic tree based on all SNPs information was drawn using FigTree version 1.1.2 (Fig. 2).

By sequencing the entire $\mathrm{pXO} 1$ and $\mathrm{pXO} 2$ DNA derived from BA103 and BA104 and comparing them with the nucleotide sequences of two plasmids of 17 other strains, 235 and 177 SNPs were identified on pXO1 and pXO2 plasmids, respectively (data not shown). The unrooted phylogenetic trees constructed by the extracted SNPs (Fig.2) revealed that $\mathrm{BA} 103$ was clustered into the $\mathrm{A} 3 \mathrm{~b}$ together with the Ames Ancestor and A2012, while BA104 in the A3a with the Australia94. The result that phylogenetic relationships disclosed by the MLVA25 and SNP analysis were quite similar strongly suggested that the SNP analysis devised here as well as MLVA25 had very high resolution power in terms of discrimination of B. anthracis strains. Furthermore, the results obtained by the SNP analysis using pXO1 or pXO2 sequence data alone coincided with that obtained by using chromosomal DNA sequences with 17 foreign strains from database (5684 SNPs, data not shown), suggesting that the identification of $B$. anthracis at the strain level would be feasible if the nucleotide sequence of either plasmid DNA is available (Fig. 2).

In this study, it was shown that either MLVA25 or SNP could be applied for the identification of $B$. anthracis at the strain level and that Japanese strains were grouped into the $\mathrm{A} 3 \mathrm{a}$ and $\mathrm{A} 3 \mathrm{~b}$ cluster. Since the strains analyzed in this study were derived from regions or countries outside of Asia, it is necessary to include Asian strains in the phylogenetic analysis with high discrimination power such as MLVA25 or SNPs analysis to understand the possible routes of introduction of $B$. anthracis strains to Japan.

We thank Drs. Masanao Matayoshi and Hirokazu Motonaga of Okinawa prefectural Livestock Sanitation Measures Office for providing us with $B$. anthracis strains isolated in Okinawa prefecture. We are also indebted to the staffs of the Miyagi Prefectural Institute of Public Health and Environment, Mie Prefecture Health and Environment Research Institute, Shiga Livestock Hygiene Service Center, Shizuoka To-bu Meat Inspection Office, and Hamamatsu City Institute for Hygiene and Environment for providing other clinical isolates.

\section{REFERENCES}

1. Amano, H., Kajio, N., Mizoguchi, T., Ohmura, K., Sone, M. and Tsuchiya, Y. 1987. Pathological observation on intestinal anthrax in swine. J. Jpn. Vet. Med. Assoc. 40: 124.

2. Anaraki, S., Addiman, S., Nixon, G., Krahé, D., Ghosh, R., Brooks, T., Lloyd, G., Spencer, R., Walsh, A., McCloskey, B. and Lightfoot, N. 2008. Investigations and control measures following a case of inhalation anthrax in East London in a drum maker and drummer, Eurosurveillance 13.

3. Gordon, D., Abajian, C. and Green, P. 1998. Consed: a graphical tool for sequence finishing. Genome Res. 8: 195-202.

4. Keim, P., Kalif, A., Schupp, J., Hill, K., Travis, S. E., Richmond, K., Adair, D. M., Hugh-Jones, M., Kuske, C. R. and Jackson, P. 1997. Molecular evolution and diversity in Bacillus anthracis as detected by amplified fragment length polymorphism markers. J. Bacteriol. 179: 818-824.

5. Keim, P., Price, L. B., Klevytska, A. M., Smith, K. L., Schupp, J. M., Okinaka, R., Jackson, P. J. and Hugh-Jones, M. E. 2000. Multiple-locus variable-number tandem repeat analysis reveals genetic relationships within Bacillus anthracis. J. Bacteriol. 182: 2928-2936.

6. Lista, F., Faggioni, G., Valjevac, S., Ciammaruconi, A., Vaissaire, J., le Doujet, C., Gorge, O., De Santis, R., Carattoli, A., Ciervo, A., Fasanella, A., Orsini, F., D’Amelio, R., Pourcel, C., Cassone, A. and Vergnaud, G. 2006. Genotyping of Bacillus anthracis strains based on automated capillary 25-loci multiple locus variable-number tandem repeats analysis. BMC Microbiol. 6: 33 .

7. Makino, S., Uchida, I., Terakado, N., Sasakawa, C. and Yoshikawa, M. 1989. Molecular characterization and protein analysis of the cap region, which is essential for encapsulation 
in Bacillus anthracis. J. Bacteriol. 171: 722-730.

8. Ministry of Agriculture, Forestry and Fisheries, Tokyo, Japan. 1982. Statistics on Animal Hygiene, pp. 14-15. In: Livestock Industry Bureau (ed.).

9. Ministry of Agriculture, Forestry and Fisheries, Tokyo, Japan. 2000. Statistics on Animal Hygiene, pp. 16-17. In: Livestock Industry Bureau (ed.).

10. Natori, N. 1995. A case of cutaneous anthrax. Tohoku J. Exp. Med. 176: 187-190.

11. Okinaka, R. T., Cloud, K., Hampton, O., Hoffmaster, A. R., Hill, K. K., Keim, P., Koehler, T. M., Lamke, G., Kumano, S., Mahillon, J., Manter, D., Martinez, Y., Ricke, D., Svensson, R. and Jackson, P. J. 1999. Sequence and organization of pXO1, the large Bacillus anthracis plasmid harboring the anthrax toxin genes. J. Bacteriol.181: 6509-6515.

12. Read, T. D., Salzberg, S. L., Pop, M., Shumway, M.,
Umayam, L., Jiang, L., Holtzapple, E., Busch, J. D., Smith, K. L., Schupp, J. M., Solomon, D., Keim, P. and Fraser, C. M. 2002. Comparative genome sequencing for discovery of novel polymorphisms in Bacillus anthracis. Science 296:2028-2033.

13. Song, J., Xu, Y., White, S., Miller, K. W. and Wolinsky, M. 2005. SNPsFinder--a web-based application for genome-wide discovery of single nucleotide polymorphisms in microbial genomes. Bioinformatics 21: 2083-2084.

14. Van Ert, M. N., Easterday, W. R., Huynh, L. Y., Okinaka, R. T., Hugh-Jones, M. E., Ravel, J., Zanecki, S. R., Pearson, T., Simonson, T. S, U'Ren, J. M., Kachur, S. M., Leadem-Dougherty, R. R., Rhoton, S. D., Zinser, G., Farlow, J., Coker, P. R., Smith, K. L., Wang, B., Kenefic, L. J., Fraser-Liggett, C. M., Wagner, D. M. and Keim, P. 2007. Global genetic population structure of Bacillus anthracis. PLoS ONE 2: e461. 